\title{
The target therapy of ovarian clear cell carcinoma
}

This article was published in the following Dove Press journal:

OncoTargets and Therapy

23 September 2014

Number of times this article has been viewed

Ying Jin

Yan Li

Lingya Pan

Department of Obstetrics and Gynecology, Peking Union Medical College Hospital, Chinese Academy of Medical Sciences and Peking Union Medical College, Beijing, People's Republic of China
Correspondence: Lingya Pan Department of Obstetrics and Gynecology, Peking Union Medical College Hospital, Chinese Academy of Medical Sciences and Peking Union Medical College, No I Shuai Fu Yuan, Dongcheng District, Beijing 100730,

People's Republic of China

Tel +861069155731

Fax +861069158274

Email lingyapan@hotmail.com
Abstract: Clear cell adenocarcinoma (CCC) of the ovary accounts for $10 \%$ of epithelial ovarian cancer and is a distinct entity from other epithelial ovarian carcinomas. It arises from the endometriosis. CCC has specific biological and clinical behavior. Compared with other histological types, CCC shows a chemoresistant phenotype, which leads to poorer prognosis. Thus, development of new target-based therapies remains an unmet need for these patients. Mutations in the gene ARIDIA have been found to occur in high frequency in CCC. The majority of these mutations lead to a loss of expression of the ARID1A protein, which is a subunit of the SWItch/ Sucrose NonFermentable (SWI/SNF) chromatin remodeling complex and considered as a bona fide tumor suppressor. Upregulation of the PIK3/AKT/mTOR pathway, particularly through mutations of PIK3CA and inactivation of PTEN, is involved in tumorigenesis of CCC. Targeting angiogenesis, the Met protooncogene pathway, and HER2 are also discussed in this review.

Keywords: ARID1A, PIK3/AKT/mTOR pathway, angiogenesis, Met protooncogene pathway, HER2

\section{Introduction}

Epithelial ovarian cancer (EOC) is the most lethal gynecologic malignancy. A majority of cases (61\%) are diagnosed at an advanced stage, with a corresponding 5-year survival rate of only $27 \%{ }^{1}$ Clear cell adenocarcinoma (CCC) accounts for $10 \%$ of EOC 2 and is a distinct entity from other epithelial ovarian carcinomas. Unlike serous EOC, which is thought to originate in the distal fallopian tube, ${ }^{3}$ the CCCs and endometrioid carcinoma were thought to arise from the endometriosis. A large epidemiological pooled analysis of 13 case-control studies, including 13,226 controls and 7,911 women with invasive ovarian cancer, investigated the frequency of self-reported endometriosis and found significantly increased risks for ovarian cancer in women with a history of endometriosis, with an odds ratio of 1.46 (95\% confidence interval [CI] 1.31-1.63, $P<0.0001)$. Importantly, a significant association of the histological subtypes of clear cell (odds ratio [OR] 3.05, 95\% CI 2.43-3.84, $P<0.0001$ ), endometrioid (OR 2.04, 95\% CI 1.67-2.48, $P<0.0001$ ), and low-grade serous ovarian carcinomas (OR 2.11, $95 \%$ CI 1.39-3.20, $P<0.0001)$ was found with endometriosis in this study. In contrast, there was no association of endometriosis with mucinous and high-grade serous cancer as well as borderline tumors. ${ }^{4}$

$\mathrm{CCC}$ has specific biological and clinical behavior. Compared with other histological types, CCC has been recognized to show a chemoresistant phenotype, leading to poorer prognosis. The response rate by paclitaxel plus carboplatin was lower than the other histologic type, ranging from $22 \%$ to $56 \%$. Thus, development of new target-based 
therapies remains an unmet need for these patients. This review discusses molecular characteristics of $\mathrm{CCC}$, frequent mutations and pathways involved, and potential implications of these factors on choice of therapy.

\section{Targeting ARIDIA}

ARIDIA gene mutations are the most frequent mutations in CCC. ARIDIA somatic mutations were seen in 46\%-57\% of ovarian clear cell carcinomas, $30 \%$ endometrioid carcinomas, and none of the high-grade serous ovarian carcinomas. ARID1A encodes BAF250a, a key component of the SWItch/ Sucrose NonFermentable (SWI/SNF) chromatin remodeling complex. Loss of the BAF250a protein correlated strongly with the ovarian clear cell carcinoma and endometrioid carcinoma subtypes. ARID1A mutations and loss of BAF250a expression were also evident in the tumor and contiguous atypical endometriosis but not in distant endometriotic lesions. ${ }^{5,6}$ Recently, Katagiri et al $^{7}$ reported ARID1A protein expression by immunohistochemistry in 60 surgically-treated ovarian CCC patients. Loss of ARID1A expression was identified in nine (15\%) of 60 samples. Loss of ARID1A in ovarian clear cell carcinoma is a negative prognostic factor in patients treated with platinum-based chemotherapy. Loss of ARID1A correlated with shorter progression-free survival (PFS) and overall survival and chemoresistance.

The ARIDIA gene encodes the adenine-thymine-rich interacting domain containing protein 1A (ARID1A), also known as BAF250a, which belongs to the SWI/SNF chromatin remodeling family and contains a 100-amino acid DNAbinding ARID domain, that binds in a sequence nonspecific manner to DNA. ${ }^{8}$ The complex has a major role in the repair of DNA lesions directly by facilitating DNA accessibility on the chromatin or indirectly by facilitating the functions of DNA repair proteins such as p53, BRCA1, GADD45, and Fanconi anemia proteins. ${ }^{9}$ The SWI/SNF complex is increasingly being recognized as a tumor-suppressor complex, with mutations in a number of subunits identified in a variety of malignancies including bladder transitional cell carcinoma, ${ }^{10}$ breast cancer, ${ }^{11}$ and pancreatic cancer. ${ }^{12}$

The mechanism relating tumorigenesis to ARID1A mutations is unclear. Yamamoto et $\mathrm{al}^{13}$ report that among the precursor lesions adjacent to the 23 ARID1A-deficient carcinomas, $86 \%$ of the nonatypical endometriosis, $100 \%$ of the atypical endometriosis, benign and borderline clear cell adenofibroma components were found to be ARID1A deficient. In contrast, in the patients with ARID1Aintact carcinomas, all of the adjacent precursor lesions retained ARID1A expression, regardless of their type and cytological atypia. The results suggest that loss of ARID1A protein expression occurs as a very early event in ovarian clear cell carcinoma development. Furthermore, tumors with ARIDIA mutations also frequently harbor PTEN or PIK3CA mutations, suggesting their collaboration in tumorigenesis. ${ }^{13}$ A recent report demonstrated that inactivation of ARID1A alone is insufficient for tumor initiation; it requires additional genetic alteration(s) such as PTEN deletion to drive tumorigenesis. ${ }^{14}$ Another gene expression analysis identified several downstream targets of ARID1A including $C D K N 1 A$ and $S M A D 3$, which are well-known p53 target genes. CDKN1A (encoding $\mathrm{p} 21$ ) acted in part to mediate growth suppression by ARID1A. The ARID1A/BRG1 complex interacted directly with p53, and mutations in the ARID1A and TP53 genes were mutually exclusive in the tumor specimens examined. ARID1A is a bona fide tumor suppressor that collaborates with p53 to regulate $C D K N 1 A$ and SMAD3 transcription and tumor growth in CCC and other endometriosis-related ovarian cancers. ${ }^{15}$

It can be speculated that once the mechanisms of the cellular dysfunctions caused by ARIDIA mutation in CCCs are accurately demonstrated, they can be explored for the development of novel targeted therapies.

\section{Targeting the PI3K/AKT/mTOR signaling pathway}

The PI3K/Akt/mTOR pathway is a central regulator in both normal cell physiology and in cancer proliferation, tumorigenesis, and metastasis. The pathway is comprised of three main driving molecules: PI3 kinase (PI3K), AKT, and mammalian target of rapamycin (mTOR). The pathway is frequently abnormal in CCC, including mutations in PIK3CA, deletion in PTEN, amplification of $A K T 1, A K T 2$, and $A K T 3$, which all lead to an aberrant functioning PI3K/AKT/mTOR pathway.

Mutations of PIK3CA (which encodes p110a), resulting in overactivation of PI3K kinase activity, have been identified in $33 \%$ of CCC patients ${ }^{16}$ and are present in the endometriosis of patients with CCC harboring PIK3CA mutations, ${ }^{17}$ indicating that the $\mathrm{PI} 3 \mathrm{~K}$ pathway has an important role in the pathogenesis of CCC, and PIK3CA mutation is an early event in the development of endometriosis-associated ovarian CCC. In patients with a $P I K 3 C A$ activating mutation, $40 \%$ also had an inactivating mutation in the regulatory genes $P I K 3 R 1$ or PTEN. ${ }^{18}$ Loss of PTEN is a coregulator of PI3K activity and is sufficient in conjunction with a mutant $P I K 3 C A$ for tumorigenesis to occur. ${ }^{18}$

AKT is the central molecule in the PI3K/AKT/ mTOR pathway, activating and modulating numerous 
downstream targets. $A K T 2$ amplification has been found in a subgroup of CCCs that are associated with a shorter PFS. ${ }^{19}$

$\mathrm{mTOR}$ is a $289 \mathrm{kDa}$ serine/threonine kinase that belongs to the PI3K-related protein kinase family. An immunohistochemical analysis of phospho-mTOR expression in a tissue microarray of 98 primary ovarian cancers showed that mTOR was more frequently activated in CCC than in serous carcinomas $(86.6 \%$ versus $50 \%) .{ }^{20}$ Another study also confirmed mTORC2 was more frequently activated in CCCs than in serous carcinomas $(71.2 \%$ versus $45.7 \%){ }^{21}$

Given that the PI3K/AKT/mTOR signaling pathway is more frequently activated in $\mathrm{CCCs}$, it is reasonable to act as a therapeutic target. Many $\mathrm{PI} 3 \mathrm{~K} / \mathrm{AKT} / \mathrm{mTOR}$ pathway inhibitors have been used in preclinical ovarian cancer models. For example, PI3K inhibitor CH513279922 and AKT inhibitor MK2206 23 have been reported to inhibit cell growth in ovarian cancer cell lines. The mTOR inhibitor everolimus can also markedly inhibit cell growth in both cisplatin-sensitive parental and cisplatin-resistant human CCC cell lines. ${ }^{20}$ However, the sensitivity of CCC cell lines to BEZ235 or temsirolimus (both are PI3K/mTOR dual inhibitors) was not related to the mutation status, but CCC cells exhibited G1 phase arrest and apoptosis with a higher concentration of BEZ235. BEZ235 significantly inhibited tumor growth in mice bearing OVISE and TUOC- 1 cell tumors. ${ }^{24}$ Another study revealed that BEZ235 prevents epithelial-mesenchymal transition induced by hypoxia and transforming growth factor- $\beta 1 .{ }^{25}$ Dual PI3K/ mTOR inhibitor DS-7423 was recently reported to constitute a promising molecular targeted therapy for CCC, and its antitumor effect might be partly obtained by induction of TP53-dependent apoptosis in TP53 wild-type CCCs. ${ }^{26}$

The majority of the agents targeting the PI3K pathway are in early-phase clinical trials, and to date, there is no evidence that supports their use in the management of CCCs. An international collaborative study led by the Gynecologic Oncology Group is evaluating the combination of the mTOR inhibitor temsirolimus with carboplatin and paclitaxel for the first-line treatment of patients with advanced CCCs. ${ }^{27}$

\section{Targeting angiogenesis}

Growth factors have been identified to play key roles in driving angiogenesis and, thus, the formation of new blood vessels that assist in "feeding" cancer. Such molecules include vascular endothelial growth factor (VEGF), platelet derived growth factor (PDGF), fibroblast growth factor, and angiopoietin/Tie2 receptor complex. VEGF has emerged as an important therapeutic target in several solid tumors, including ovarian carcinoma.
The monoclonal antibody bevacizumab binds VEGF, thus preventing activation of the VEGF receptor (VEGFR), leading to inhibition of tumor angiogenesis. Four clinical trials including first-line trials of the International Collaborative Ovarian Neoplasm Group trial (ICON7), the Gynecologic Oncology Group trial (GOG218), and the trials in recurrent cases of Ovarian Cancer Study Comparing Efficacy and Safety of Chemotherapy and Anti-Angiogenic Therapy in Platinum-Sensitive Recurrent Disease (OCEANS) and Avastin Use in Platinum-Resistant Epithelial Ovarian Cancer (AURELIA) have shown that the tumor vasculature is a valid target. ICON7 and GOG218 showed improvements in PFS, and in the case of ICON7, increased overall survival in a predefined group of patients at high risk of disease progression. OCEANS and AURELIA also showed improvements in $\mathrm{PFS},{ }^{28}$ but response rates in specific histological subgroups were not examined.

Mabuchi et $\mathrm{al}^{29}$ examined the role of VEGF as a therapeutic target in CCC. Immunohistochemical analysis using tissue microarrays of 98 primary ovarian cancers revealed that VEGF was strongly expressed both in early stage and advanced stage CCC. In early stage CCCs, patients who had tumors with high levels of VEGF had significantly shorter survival than those with low levels of VEGF. Furthermore, in vitro experiments revealed that VEGF expression was significantly higher in cisplatin-refractory human CCC cells compared with the respective parental cells. In vivo treatment with bevacizumab markedly inhibited the growth of both parental CCC cell-derived and cisplatin-refractory CCC cell-derived tumors. VEGF expression has been shown to be upregulated by factors such as insulin-like growth factor- 1 and interleukin (IL)-6. ${ }^{30}$ Anglesio et $\mathrm{al}^{31}$ found specific overexpression of the IL6-STAT3-HIF (IL-6-signal transducer and activator of transcription 3-hypoxia induced factor) pathway in CCC tumors compared with high-grade serous cancers. The authors also reported sustained clinical and functional imaging responses in two CCC patients with chemotherapy-resistant disease who were treated with sunitinib, a potent inhibitor of several tyrosine kinases including VEGFR, platelet-derived growth factor receptor, and c-Kit, which has significant activity in renal clear cell cancer. ${ }^{32}$ A study by Stany et $\mathrm{al}^{33}$ also confirmed the use of sunitinib in CCC. They demonstrated that clear cell tumors are exquisitely sensitive to sunitinib compared with serous tumors. There are two international collaborative studies to evaluate efficacy of sunitinib for the treatment of persistent or recurrent CCC. ${ }^{34,35}$ A case report of chemoresistant $\mathrm{CCC}^{36}$ confirmed the use of combination therapy with sorafenib, a multikinase inhibitor that targets mitogen-activated protein kinase or 
the Ras/Raf/ERK pathway and also inhibits other kinases (VEGFR, platelet-derived growth factor receptor). ${ }^{37}$

\section{Targeting the Met protooncogene pathway}

Met protooncogene (also called hepatocyte growth factor receptor or MET) encodes MET kinase, which is composed of three functional domains including the ligand-binding domain, regulatory juxtamembrane domain, and the receptor tyrosine kinase domain. When its ligand, hepatocyte growth factor, binds, the Ras-mitogen-activated protein kinase pathway is activated as well as the phosphatidylinositol 3-kinase-AKTmTOR signaling pathway and several other pathways. ${ }^{38,39} \mathrm{In}$ tumor cells, MET can be activated in a ligand-independent manner through amplification and overexpression of the MET gene. MET amplification was identified in $4 \%-10 \%$ of gastric cancers, esophageal cancers, lung cancers, and colorectal cancers. ${ }^{40-43}$ MET overexpression and gene amplification were commonly detected in CCC with their frequencies at $22 \%$ and $24 \%$, respectively. Such alterations were extremely rare in the other histological subtypes of ovarian carcinoma (ie, serous, endometrioid, and mucinous adenocarcinomas). MET overexpression was associated with a worse prognosis in the CCC patients. ${ }^{44}$ Furthermore, in the case with synchronous endometriosis and with adjacent CCC components, the overall incidence of MET overexpression gradually increased from the precursors of nonatypical form $(0 \%)$, through those of atypical form $(67 \%)$ and the relatively differentiated carcinoma components $(92 \%)$, to the poorly differentiated carcinoma components $(100 \%)$. The results indicated that MET alterations occur as an early event in carcinogenesis of the MET amplification-positive CCC, and these alterations might drive the development and progression in a subset of CCC ${ }^{45}$ Furthermore, Yamashita et a ${ }^{46}$ also revealed $M E T$ gene amplification in four out of 13 CCC primary tumors and two out of eight $\mathrm{CCC}$ cell lines using an array-based comparative genomic hybridization analysis. A total of $37 \%$ demonstrated $M E T$ gene amplifications (more than four copies) were confirmed in $73 \mathrm{CCC}$ cases using real-time quantitative polymerase chain reaction. In the meantime, amplification of the AKT2 gene was also observed in five out of 21 samples by array-based comparative genomic hybridization analysis. MET knockdown in CCC cell lines with MET amplification showed both increased apoptosis and senescence in vitro, which suggested that $M E T$ amplified CCC cells primarily depend on activation of the MET/PI3K/AKT pathway for cell proliferation and survival. Targeted therapy inhibiting the MET/PI3K/AKT pathway may be promising for $\mathrm{CCC}$ treatment.

\section{HER2 as a therapeutic target}

Tan et $\mathrm{al}^{19}$ reported that HER2 gene amplification and protein overexpression were observed in $14 \%$ of CCCs using hierarchical cluster analysis. Using an immunohistochemical method, Fujimura et al reported that HER2 was frequently overexpressed in $42.9 \%$ of CCC patients compared with $20.8 \%$ of ovarian serous adenocarcinoma, $23.1 \%$ of ovarian endometrioid adenocarcinoma, and $30.0 \%$ of mucinous adenocarcinoma specimens. Trastuzumab, a humanized recombinant monoclonal antibody against HER2, significantly and dose-dependently reduced the growth of CCC cell lines in vitro. Trastuzumab also dose-dependently inhibited the growth of xenografted RMG-1 (CCC cell line) tumor. The survival period of trastuzumab-treated mice was longer than that of the control group. ${ }^{47}$ Thus, trastuzumab appears to be a candidate treatment modality for HER2 overexpressing CCC. A clinical trial was conducted to use single-agent trastuzumab to treat HER2-overexpression ovarian and primary peritoneal carcinoma (documented by an immunohistochemical method). ${ }^{48}$ An overall response rate of only $7 \%$ was observed. However, patient selection (41 patients of which seven were CCCs) was based only on HER2 immunohistochemical expression levels, without assessment of HER 2 copy number status. Future studies are warranted to assess the predictive value of HER 2 amplification and overexpression in CCCs.

The distinct molecular features of CCC and serous ovarian cancer serve to emphasize the need to develop subtypespecific therapeutic approaches in the management of EOC. Patients with CCC have poor prognosis and short survival due to lack of effective therapy. Despite displaying histologically uniform features, CCCs do not constitute a single entity and may be classified into distinct molecular genetic subtypes that also appear to be associated with clinical outcome. Future research is expected to help better understand the pathogenesis of CCC and identify new compounds to block important signaling pathways.

\section{Disclosure}

The authors report no conflicts of interest in this work.

\section{References}

1. American Cancer Society. Cancer Facts and Figures, 2013 [webpage on the Internet]. Atlanta, GA: American Cancer Society; 2013. Available from: http://www.cancer.org/research/cancerfactsfigures/cancerfactsfigures/cancer-facts-figures-2013. Accessed November 6, 2013.

2. Lee KR, Tavassoli FA, Prat J, et al. Surface epithelial-stromal tumours: tumours of the ovary and peritoneum. In: Tavassoli FA, Devilee P, editors. World Health Organization Classification of Tumours: Pathology and Genetics of Tumours of the Breast and Female Genital Organs. Lyon: IARC Press; 2003:117-145. 
3. Lee Y, Miron A, Drapkin R, et al. A candidate precursor to serous carcinoma that originates in the distal fallopian tube. J Pathol. 2007;211(1): 26-35.

4. Ness RB, Grisso JA, Cottreau C, et al. Factors related to inflammation of the ovarian epithelium and risk of ovarian cancer. Epidemiology. 2000;11(2):111-117.

5. Jones S, Wang TL, Shih IeM, et al. Frequent mutations of chromatin remodeling gene ARID1A in ovarian clear cell carcinoma. Science. 2010;330(6001):228-231.

6. Wiegand KC, Shah SP, Al-Agha OM, et al. ARID1A mutations in endometriosis-associated ovarian carcinomas. $N$ Engl J Med. 2010;363(16): 1532-1543.

7. Katagiri A, Nakayama K, Rahman MT, et al. Loss of ARID1A expression is related to shorter progression-free survival and chemoresistance in ovarian clear cell carcinoma. Mod Pathol. 2012;25(2):282-288.

8. Rutgers JL, Scully RE. Ovarian mullerian mucinous papillary cystadenomas of borderline malignancy. A clinicopathologic analysis. Cancer. 1988;61(2):340-348

9. Reisman D, Glaros S, Thompson EA. The SWI/SNF complex and cancer. Oncogene. 2009;28(14):1653-1668.

10. Gui Y, Guo G, Huang Y, et al. Frequent mutations of chromatin remodeling genes in transitional cell carcinoma of the bladder. Nat Genet. 2011;43(9):875-878.

11. Mamo A, Cavallone L, Tuzmen S, et al. An integrated genomic approach identifies ARID1A as a candidate tumor-suppressor gene in breast cancer. Oncogene. 2012;31(16):2090-2100.

12. Shain AH, Giacomini CP, Matsukuma K, et al. Convergent structural alterations define SWItch/Sucrose NonFermentable (SWI/ $\mathrm{SNF}$ ) chromatin remodeler as a central tumor suppressive complex in pancreatic cancer. Proc Natl Acad Sci U S A. 2012;109(5): E252-E259.

13. Yamamoto S, Tsuda H, Takano M, Tamai S, Matsubara O. Loss of ARID1A protein expression occurs as an early event in ovarian clearcell carcinoma development and frequently coexists with PIK3CA mutations. Mod Pathol. 2012;25(4):615-624.

14. Guan B, Rahmanto YS, Wu RC, et al. Roles of deletion of Arid1a, a tumor suppressor, in mouse ovarian tumorigenesis. J Natl Cancer Inst. 2014;106(7).

15. Guan B, Wang TL, Shih IeM. ARID1A, a factor that promotes formation of SWI/SNF-mediated chromatin remodeling, is a tumor suppressor in gynecologic cancers. Cancer Res. 2011;71(21):6718-6727.

16. Kuo KT, Mao TL, Jones S, et al. Frequent activating mutations of PIK3CA in ovarian clear cell carcinoma. Am J Pathol. 2009;174(5): 1597-1601.

17. Yamamoto S, Tsuda H, Takano M, Iwaya K, Tamai S, Matsubara O. PIK3CA mutation is an early event in the development of endometriosisassociated ovarian clear cell adenocarcinoma. J Pathol. 2011;225(2): 189-194.

18. Kinross KM, Montgomery KG, Kleinschmidt M, et al. An activating Pik3ca mutation coupled with Pten loss is sufficient to initiate ovarian tumorigenesis in mice. J Clin Invest. 2012;122(2):553-557.

19. Tan DS, Iravani M, McCluggage WG, et al. Genomic analysis reveals the molecular heterogeneity of ovarian clear cell carcinomas. Clin Cancer Res. 2011;17(6):1521-1534.

20. Mabuchi S, Kawase C, Altomare DA, et al. mTOR is a promising therapeutic target both in cisplatin-sensitive and cisplatin-resistant clear cell carcinoma of the ovary. Clin Cancer Res. 2009;15(17): 5404-5413.

21. Hisamatsu T, Mabuchi S, Matsumoto Y, et al. Potential role of mTORC2 as a therapeutic target in clear cell carcinoma of the ovary. Mol Cancer Ther. 2013;12(7):1367-1377.

22. Tanaka H, Yoshida M, Tanimura H, et al. The selective class I PI3K inhibitor CH5132799 targets human cancers harboring oncogenic PIK3CA mutations. Clin Cancer Res. 2011;17(10):3272-3281.

23. Yap TA, Yan L, Patnaik A, et al. First-in-man clinical trial of the oral pan-AKT inhibitor MK-2206 in patients with advanced solid tumors. J Clin Oncol. 2011;29(35):4688-4695.
24. Oishi T, Itamochi H, Kudoh A, et al. The PI3K/mTOR dual inhibitor NVP-BEZ235 reduces the growth of ovarian clear cell carcinoma. Oncol Rep. 2014;32(2):553-558.

25. Lin G, Gai R, Chen Z, et al. The dual PI3K/mTOR inhibitor NVPBEZ235 prevents epithelial-mesenchymal transition induced by hypoxia and TGF-ß1. Eur J Pharmacol. 2014;729:45-53.

26. Kashiyama T, Oda K, Ikeda Y, et al. Antitumor activity and induction of TP53-dependent apoptosis toward ovarian clear cell adenocarcinoma by the dual PI3K/mTOR inhibitor DS-7423. PLoS One. 2014;9(2):e87220.

27. ClinicalTrials.gov. Temsirolimus, carboplatin, and paclitaxel as firstline therapy in treating patients with newly diagnosed stage III-IV clear cell ovarian cancer [webpage on the Internet]. Bethesda, MD: National Institutes of Health; 2010 [updated June 23, 2014]. Available from: http://clinicaltrials.gov/ct2/show/NCT01196429. Accessed September 2, 2014.

28. Shaw D, Clamp A, Jayson GC. Angiogenesis as a target for the treatment of ovarian cancer. Curr Opin Oncol. 2013;25(5):558-565.

29. Mabuchi S, Kawase C, Altomare DA, et al. Vascular endothelial growth factor is a promising therapeutic target for the treatment of clear cell carcinoma of the ovary. Mol Cancer Ther. 2010;9(8):2411-2422.

30. Salgado R, Benoy I, Weytjens R, et al. Arterio-venous gradients of IL-6, plasma and serum VEGF and D-dimers in human cancer. Br J Cancer. 2002;87(12):1437-1444.

31. Anglesio MS, George J, Kulbe H, et al; Australian Ovarian Cancer Study Group. IL6-STAT3-HIF signaling and therapeutic response to the angiogenesis inhibitor sunitinib in ovarian clear cell cancer. Clin Cancer Res. 2011;17(8):2538-2548.

32. Kumar R, Crouthamel MC, Rominger DH, et al. Myelosuppression and kinase selectivity of multikinase angiogenesis inhibitors. Br J Cancer. 2009;101(10):1717-1723.

33. Stany MP, Vathipadiekal V, Ozbun L, et al. Identification of novel therapeutic targets in microdissected clear cell ovarian cancers. PLoS One. 2011;6(7):e21121.

34. ClinicalTrials.gov. Sunitinib malate in treating patients with persistent or recurrent clear cell ovarian cancer [webpage on the Internet]. Bethesda, MD: National Institutes of Health; 2009 [updated August 4 2014]. Available from: http://clinicaltrials.gov/ct2/show/NCT00979992. Accessed August 4, 2014.

35. ClinicalTrials.gov. Sunitinib ${ }^{\circledR}$ in patients with recurrent ovarian clear cell carcinoma [webpage on the Internet]. Bethesda, MD: National Institutes of Health; 2013 [updated April 7, 2013]. Available from: http:// clinicaltrials.gov/ct2/show/NCT01824615. Accessed April 7, 2013.

36. Rahman M, Nakayama K, Ishibashi T, et al. A case of stage III c ovarian clear cell carcinoma: the role for predictive biomarkers and targeted therapies. Int J Mol Sci. 2013;14(3):6067-6073.

37. Han ES, Lin P, Wakabayashi M. Current status on biologic therapies in the treatment of epithelial ovarian cancer. Curr Treat Options Oncol. 2009;10(1-2):54-66.

38. Ma PC, Maulik G, Christensen J, Salgia R. c-Met: structure, functions and potential for therapeutic inhibition. Cancer Metastasis Rev. 2003;22(4):309-325.

39. Peruzzi B, Bottaro DP. Targeting the c-Met signaling pathway in cancer. Clin Cancer Res. 2006;12(12):3657-3660.

40. Hara T, Ooi A, Kobayashi M, Mai M, Yanagihara K, Nakanishi I. Amplification of c-myc, K-sam, and c-met in gastric cancers: detection by fluorescence in situ hybridization. Lab Invest. 1998;78(9): 1143-1153.

41. Miller CT, Lin L, Casper AM, et al. Genomic amplification of MET with boundaries within fragile site FRA7G and upregulation of MET pathways in esophageal adenocarcinoma. Oncogene. 2006;25(3):409-418.

42. Zhao X, Weir BA, LaFramboise T, et al. Homozygous deletions and chromosome amplifications in human lung carcinomas revealed by single nucleotide polymorphism array analysis. Cancer Res. 2005;65(13):5561-5570.

43. Di Renzo MF, Olivero M, Giacomini A, et al. Overexpression and amplification of the met/HGF receptor gene during the progression of colorectal cancer. Clin Cancer Res. 1995;1(2):147-154. 
44. Yamamoto S, Tsuda H, Miyai K, Takano M, Tamai S, Matsubara O. Gene amplification and protein overexpression of MET are common events in ovarian clear-cell adenocarcinoma: their roles in tumor progression and prognostication of the patient. Mod Pathol. 2011;24(8): $1146-1155$.

45. Yamamoto S, Tsuda H, Miyai K, Takano M, Tamai S, Matsubara O. Accumulative copy number increase of MET drives tumor development and histological progression in a subset of ovarian clear-cell adenocarcinomas. Mod Pathol. 2012;25(1):122-130.

46. Yamashita Y, Akatsuka S, Shinjo K, et al. Met is the most frequently amplified gene in endometriosis-associated ovarian clear cell adenocarcinoma and correlates with worsened prognosis. PLoS One. 2013;8(3):e57724.
47. Fujimura M, Katsumata N, Tsuda H, et al. HER2 is frequently overexpressed in ovarian clear cell adenocarcinoma: possible novel treatment modality using recombinant monoclonal antibody against HER2, trastuzumab. Jpn J Cancer Res. 2002;93(11):1250-1257.

48. Bookman MA, Darcy KM, Clarke-Pearson D, Boothby RA, Horowitz IR. Evaluation of monoclonal humanized anti-HER2 antibody, trastuzumab, in patients with recurrent or refractory ovarian or primary peritoneal carcinoma with overexpression of HER2: a phase II trial of the Gynecologic Oncology Group. J Clin Oncol. 2003;21(2):283-290.

\section{Publish your work in this journal}

OncoTargets and Therapy is an international, peer-reviewed, open access journal focusing on the pathological basis of all cancers, potential targets for therapy and treatment protocols employed to improve the management of cancer patients. The journal also focuses on the impact of management programs and new therapeutic agents and protocols on

\section{Dovepress}

patient perspectives such as quality of life, adherence and satisfaction The manuscript management system is completely online and includes a very quick and fair peer-review system, which is all easy to use. Visit http://www.dovepress.com/testimonials.php to read real quotes from published authors. 